\title{
Virology analysis in HCV genotype 1-infected patients treated with the combination of simeprevir and TMC647055/ritonavir, with and without ribavirin, and JNJ-56914845
}

\author{
Leen Vijgen ${ }^{*}$ (1), Kim Thys, An Vandebosch, Pieter Van Remoortere, René Verloes and Sandra De Meyer
}

\begin{abstract}
Background: In study TMC647055HPC2001, a 3-direct-acting-antiviral (DAA) regimen combining NS3/4A protease inhibitor simeprevir (SMV), non-nucleoside NS5B inhibitor TMC647055/ritonavir (RTV) and NS5A inhibitor JNJ-56914845 resulted in high sustained virologic response 12 weeks after actual end of treatment (SVR12) in chronic hepatitis C virus (HCV) genotype 1-infected patients. SVR12 rates were generally lower in the 2-DAA regimen SMV + TMC647055/RTV with or without ribavirin. The objective of this study was to identify and characterise pre-existing and emerging resistance-associated variants (RAVs) in patients enrolled in study TMC647055HPC2001.
\end{abstract}

Methods: HCV population sequencing analyses were performed on baseline isolates from all patients $(n=90)$ and postbaseline isolates from patients with virologic failure $(n=22)$. In addition, deep sequencing and phenotypic analyses were performed on selected baseline and post-baseline isolates.

Results: The majority of patients with virologic failure had emerging RAVs to all study drugs at the time of failure: in all 22 patients SMV RAVs emerged at NS3 positions 80,155, 156 and/or 168, consistent with the known SMV resistance profile. Emerging TMC647055 RAVs at NS5B position 495 were detected in the majority of patients (16/22), and all 5 patients who failed the 3-DAA regimen had emerging JNJ-56914845 RAVs at NS5A positions 30 and/or 31. While at the end of study emerging SMV and TMC647055 RAVs were no longer observed by population sequencing in 40\% (8/20) and 62.5\% (10/ 16) of patients with follow-up data available, respectively, emerging JNJ-56914845 RAVs were still detected in all (5/5) patients.

Conclusions: Virologic failure in the 2- and 3-DAA combinations was, in the majority of patients, associated with the emergence of RAVs to all study drugs. While emerging SMV and TMC647055 RAVs became undetectable during followup, JNJ-56914845 RAVs in NS5A were still observed at end of study.

Trial registration number: NCT01724086 (date of registration: September 26, 2012)

Keywords: Hepatitis C virus, Genotype 1, Simeprevir, TMC647055/ritonavir, JNJ-56914845

\footnotetext{
* Correspondence: Ivijgen@its.jnj.com

Janssen Research \& Development, Janssen Pharmaceutica NV, Turnhoutseweg 30, 2340 Beerse, Belgium
} 


\section{Background}

Hepatitis $\mathrm{C}$ virus ( $\mathrm{HCV}$ ) infection is a leading cause of chronic liver disease with an estimated 130-150 million people infected worldwide [1]. Since the approval in 2011 of the first direct-acting antivirals (DAAs), current therapy includes well-tolerated interferon (IFN)-free DAA combination regimens with high efficacy rates [2, 3]. For some regimens, the addition of ribavirin is indicated in certain patient populations to increase efficacy rates [4].

In the Phase 2a study TMC647055HPC2001 (NCT01724086), a 12-week 3-DAA regimen of simeprevir (SMV), TMC647055/ritonavir (RTV) and JNJ56914845 resulted in high sustained virologic response 12 weeks after actual end of treatment (SVR12; 93\% for HCV genotype [GT]1a- and $100 \%$ for GT1binfected patients in the JNJ-56914845 $60 \mathrm{mg}$ group) while SVR12 rates were lower in the 12-week 2-DAA regimens of SMV and TMC647055/RTV with or without ribavirin (RBV) (SVR12 $33-86 \%$ depending on $\mathrm{HCV}$ geno/subtype, presence of RBV and TMC647055 dose) [5].

SMV is a once-daily (QD), oral $\mathrm{HCV} \mathrm{NS3/4A} \mathrm{protease}$ inhibitor, approved with pegylated IFN (pegIFN)/RBV for the treatment of HCV GT1 and GT4 infection in the United States (US) and European Union (EU), and in IFN-free combination with sofosbuvir for GT1 infection in the US and GT1 and GT4 infection in the EU. The majority of HCV GT1-infected patients who failed $\mathrm{SMV} /$ pegIFN/RBV treatment in a Phase $2 \mathrm{~b} / 3$ clinical trial had emerging mutations at NS3 positions 80, 122, 155 and/or 168, conferring high-level resistance to SMV in vitro [6]. Similar SMV high-level resistance mutations were observed in GT4-infected patients who failed SMV/pegIFN/RBV treatment and in GT1-infected patients with virologic failure upon 12 or 24 weeks' SMV/sofosbuvir treatment [7-10]. At the end of study, these mutations could no longer be detected in half of the patients.

TMC647055 is an oral non-nucleoside inhibitor (NNI) binding at the thumb-1 NNI-1 site of the HCV NS5B polymerase [11]. The antiviral activity of TMC647055 in HCV GT1-infected patients is dosedependent, with a median maximum decrease from baseline in HCV RNA of $2.4 \log _{10} \mathrm{IU} / \mathrm{mL}$ and $3.4 \log _{10}$ $\mathrm{IU} / \mathrm{mL}$ at $1000 \mathrm{mg}$ twice daily (BID) for 5 days in GT1a- $(n=3)$ and GT1b-infected $(n=3)$ patients, respectively [12]. Emerging TMC647055 resistanceassociated variants (RAVs) at NS5B position 495 were observed in two GT1b-infected patients. Combination of TMC647055 (1000 mg BID) with SMV (150 mg QD) for 10 days in GT1-infected patients (seven GT1a and one GT1b) led to potent antiviral activity with a 4.64 $\log _{10} \mathrm{IU} / \mathrm{mL}$ median decrease in $\mathrm{HCV}$ RNA from baseline at day 11 with no viral breakthrough observed [13]. RTV was used as a pharmacokinetic enhancer to counteract cytochrome $\mathrm{P} 450 \quad 3 \mathrm{~A} 4$ induction by TMC647055, therefore boosting circulating plasma concentrations of TMC647055 and SMV when given in combination in study TMC647055HPC2001.

JNJ-56914845 (previously GSK2336805) is an oral inhibitor of HCV replication targeting the NS5A replication complex [14]. Single-dose administration of JNJ56914845 to HCV GT1-infected patients (15 GT1a; two GT1b) resulted in rapid reductions in HCV RNA, with a mean decrease in HCV RNA from baseline within the first 24 hours of $2.18 \log _{10} \mathrm{IU} / \mathrm{mL}$ for $30 \mathrm{mg}$ (one GT1a) and $2.84 \log _{10} \mathrm{IU} / \mathrm{mL}$ for $60 \mathrm{mg}$ (four GT1a; one GT1b) [15]. Emerging JNJ-56914845 RAVs were detected in 13/23 patients at NS5A positions 28, 30, 31, 32 and/or 93.

The objective of this study was to identify and characterise pre-existing and emerging RAVs in patients enrolled in study TMC647055HPC2001.

\section{Methods \\ Study design}

The TMC647055HPC2001 study design is shown in Fig. 1 [5].

\section{HCV geno/subtype determination}

$\mathrm{HCV}$ geno/subtypes were determined at screening by the VERSANT ${ }^{\odot}$ HCV Genotype 2.0 (LiPA v2.0) or, if failed, by the Trugene HCV Genotyping assay (Siemens Healthcare Diagnostics, IL, USA). HCV geno/subtypes were determined pretreatment by sequencing an NS5B 329-bp region followed by basic local alignment search tool analysis. The results of the NS5B-based assay or, if missing, from the LiPA v2.0 or Trugene assay were used for virology analyses.

\section{HCV NS3/4A, NS5B and NS5A sequence analysis}

The NS3/4A region or the NS3 protease, NS5B and NS5A gene were sequenced using population sequencing in baseline samples from all patients and postbaseline samples from patients with virologic failure [16] (see Additional file 1). In addition, Illumina deep sequencing was performed for a selection of samples (from Panels 1-2 and Panel 4), as described earlier [17]. Deep sequencing analysis was performed using a detection threshold of $1 \%$. Sequencing resulted in an average coverage of 19,572 reads (range 7,177-36,508) per amino acid position in NS3 (amino acid position 1-181), of 20,594 reads (range 463-78,381) per amino acid position in NS5B, and of 23,045 reads (range 2,563-78,342) per amino acid position in NS5A. Baseline polymorphisms were defined as amino acid changes from the H77 (GenBank accession number AF009606) or the HCV Con1 (GenBank accession 


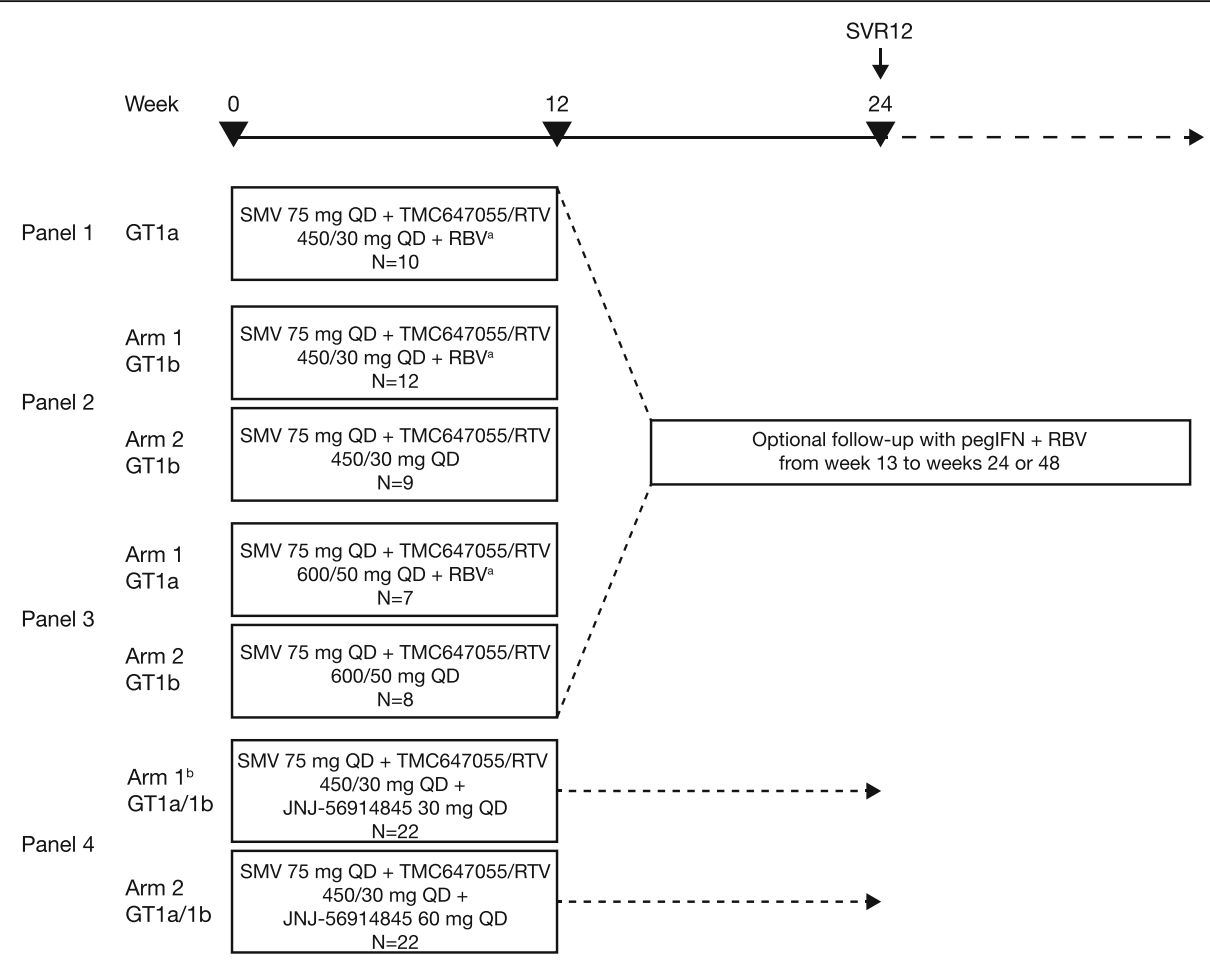

Fig. 1 TMC647055HPC2001 study design. ${ }^{a}$ Ribavirin given BID at doses of 1000-1200 mg. ${ }^{\text {bPanel }} 4$ Arm 1 included 1 GT1c-and 1 GT1/-infected patient, based on NS5B-based geno/subtyping. Follow-up therapy with pegIFN + RBV was based on on-treatment response and was initiated only if: week $4 \mathrm{HCV}$ RNA $\geq 25 \mathrm{IU} / \mathrm{mL}$ (Panels 1-3: 36 weeks of follow-up therapy); week $4 \mathrm{HCV}$ RNA <25 IU/mL detectable or HCV RNA confirmed detectable between week 4 and week 11 (Panels 1-2: 12 weeks of follow-up therapy). BID: twice daily; GT: genotype; pegIFN: pegylated interferon; QD: once daily; RBV: ribavirin; RTV: ritonavir; SMV: simeprevir; SVR12: sustained virologic response 12 weeks after actual end of treatment

number AJ238799) reference sequences for $\mathrm{HCV}$ GT1a and GT1b, respectively. Emerging mutations were defined as amino acid changes from patientspecific baseline sequences based on population sequencing. RAVs were defined as amino acid substitutions with a fold change (FC) in 50\% effective concentration $\left(\mathrm{EC}_{50}\right)>2.0$ for the respective drug, when tested as a sitedirected mutant (SDM) in a transient replicon assay.

\section{Phenotypic characterisation using a transient replicon assay}

SDMs were engineered in a GT1a or GT1b replicon. For the chimeric replicon assay, sequences of the NS3 protease domain (aa7-192), NS5B polymerase (full length) or NS5A (full length) from patient isolates were introduced in a GT1b replicon backbone generating chimeric replicons [18-21]. The NS5A chimeric replicon assay was performed at Monogram Bioscience Inc., LabCorp, San Francisco, CA, USA. Antiviral activity of the inhibitors against the NS3 protease and NS5B chimeric replicons was assessed in a transient replicon assay using luciferase read-out, as described earlier [22]. $E_{50}$ values were determined and compared with the $\mathrm{EC}_{50}$ of a reference GT1b wild-type replicon to calculate the $\mathrm{FC}$ in $\mathrm{EC}_{50}$ values.

\section{Ethical approval}

The study was approved by the Institutional Review Board or Independent Ethics Committee at each participating centre, and met the ethical principles of the Declaration of Helsinki and Good Clinical Practice guidelines. All patients provided written, informed consent.

\section{Results}

\section{Baseline polymorphisms}

At baseline, SMV RAVs were observed in 6/89 GT1infected patients (6.7\%) with NS3 sequencing data available. These included Q80K (in 5/89 GT1 [5.6\%]; 4/ 44 GT1a [9.1\%]; 1/44 GT1b [2.3\%]) and Q80R (in 1/89 GT1 [1.1\%]) (Fig. 2). Two out of the four GT1ainfected patients with baseline Q80K were treated with the 2-DAA regimen in Panel 1 and did not achieve SVR. The two other GT1a-infected patients with baseline Q80K received the 3-DAA regimen in Panel 4; one patient achieved SVR while the other experienced viral relapse. The GT1b-infected patient with Q80R at baseline had detectable HCV RNA at the end of treatment and did not achieve SVR. TMC647055 RAVs were not observed at baseline. In Panel 4, JNJ-56914845 RAVs were detected in 2/42 (4.8\%) GT1-infected patients 


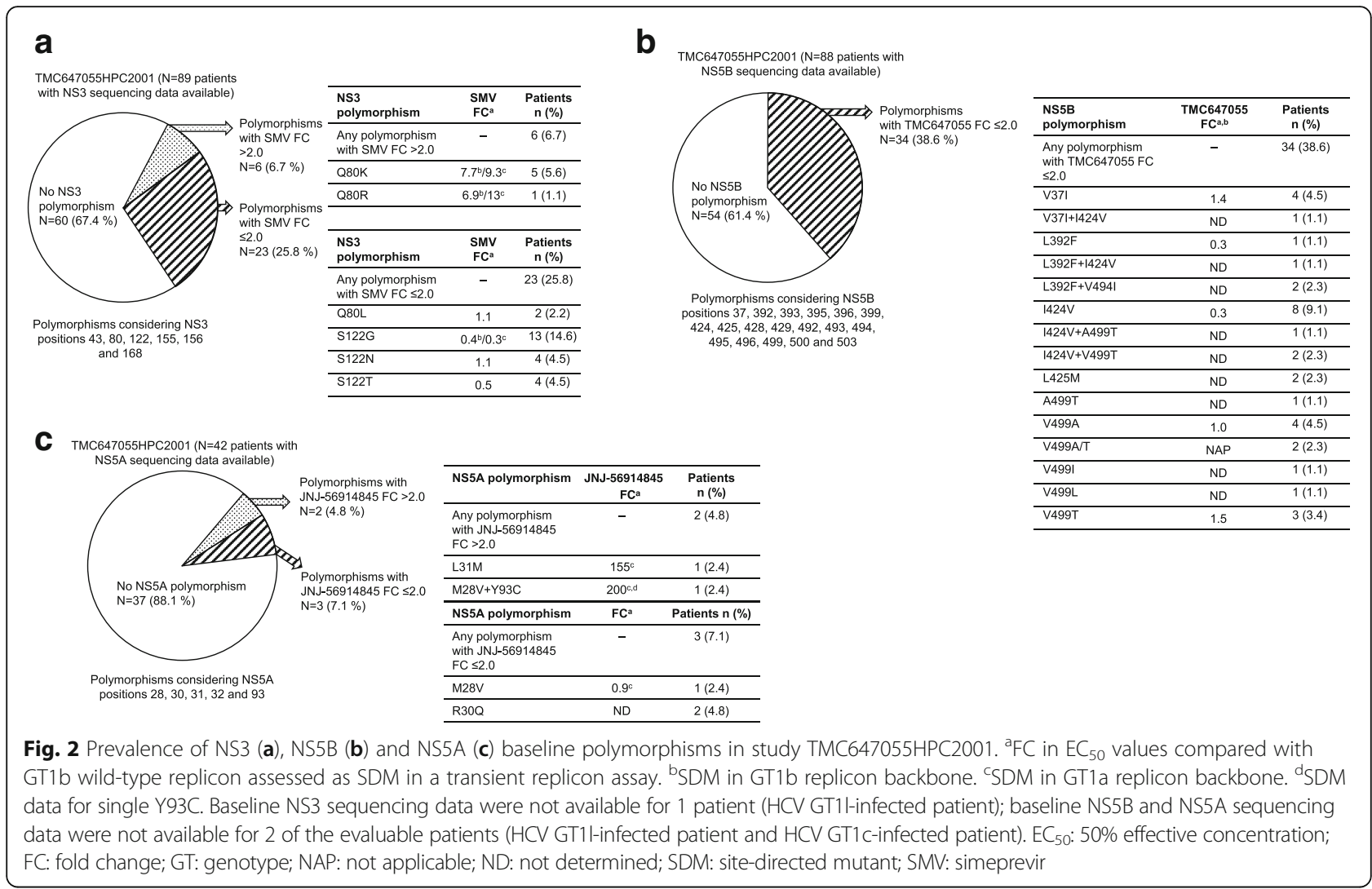

with NS5A sequencing data available; L31M and Y93C (in combination with $\mathrm{M} 28 \mathrm{~V}$ ), respectively (both in $1 / 27$ GT1a [3.7\%]). Both patients with baseline JNJ56914845 RAVs achieved SVR.

In the phenotypic analysis of a subset of baseline isolates (Fig. 3), low-level resistance to SMV was observed for four GT1a isolates with SMV RAV Q80K (median $\mathrm{FC}$ in $\mathrm{EC}_{50}=8.5$; range 5.6-11). All baseline isolates remained fully susceptible to TMC647055 (FC in $\left.\mathrm{EC}_{50} \leq 2.0\right)$. One $\mathrm{GT} 1$ a baseline isolate with NS5A RAV L31M displayed a 150-fold reduction in susceptibility to JNJ-56914845. For the baseline isolate with NS5A Y93C (+M28V), which was present as mixture with wild-type, the $\mathrm{FC}$ in $\mathrm{EC}_{50}$ was $\leq 2.0$.

\section{Emergence of RAVs}

Emergence of RAVs was investigated using population sequencing in the 22 patients with virologic failure (viral breakthrough, detectable HCV RNA at end of treatment [without viral breakthrough] or viral relapse [before or after follow-up week 12]) (Table 1).

In $14 / 17$ patients $(82.4 \%)$ with virologic failure in the 2-DAA regimen, emerging RAVs to both SMV and TMC647055 were detected at time of failure. SMV RAVs at NS3 positions 80, 155, 156 and/or 168 were observed in all 17 patients. The majority (9/10) of GT1b-infected patients had an emerging mutation at NS3 position 168 , while either an emerging R155K (alone or in combination with a Q80R) (in 4/7) or an emerging mutation at NS3 position 168 (in 3/7) was observed in the GT1a-infected patients. Emerging TMC647055 RAVs at NS5B position 495 were detected in $14 / 17$ patients, and included mainly $\mathrm{P} 495 \mathrm{~L}$ (in $11 / 14$ ).

In all five patients with virologic failure in the 3DAA regimen (all GT1a), emerging RAVs to both SMV and JNJ-56914845 were observed at time of failure. SMV RAVs emerged at NS3 positions 80 or 155; emerging JNJ-56914845 RAVs were detected at NS5A positions 30 and/or 31 (Q30E [ $n=2]$, Q30R [ $n=1]$, $\mathrm{L} 31 \mathrm{M}[n=1]$ and $\mathrm{Q} 30 \mathrm{H}+\mathrm{L} 31 \mathrm{M}[n=1])$. Emerging TMC647055 RAVs were observed in 2/5 patients, and involved P495L in both.

Illumina deep sequencing data were available for a subset of time of failure isolates (Table 1). In the three patients in Panel 4 without emerging TMC647055 RAVs at time of failure based on population sequencing, no RAVs could be detected by deep sequencing. Additional RAVs at a read frequency $\leq 25 \%$ were detected by deep sequencing in three patients with RAVs detected by population sequencing (patients $8,18,20$ ). Deep sequencing identified additional emerging RAVs at a read frequency $>25 \%$ in two patients at time of failure (patients 1, 2). RAVs emerging at time of 


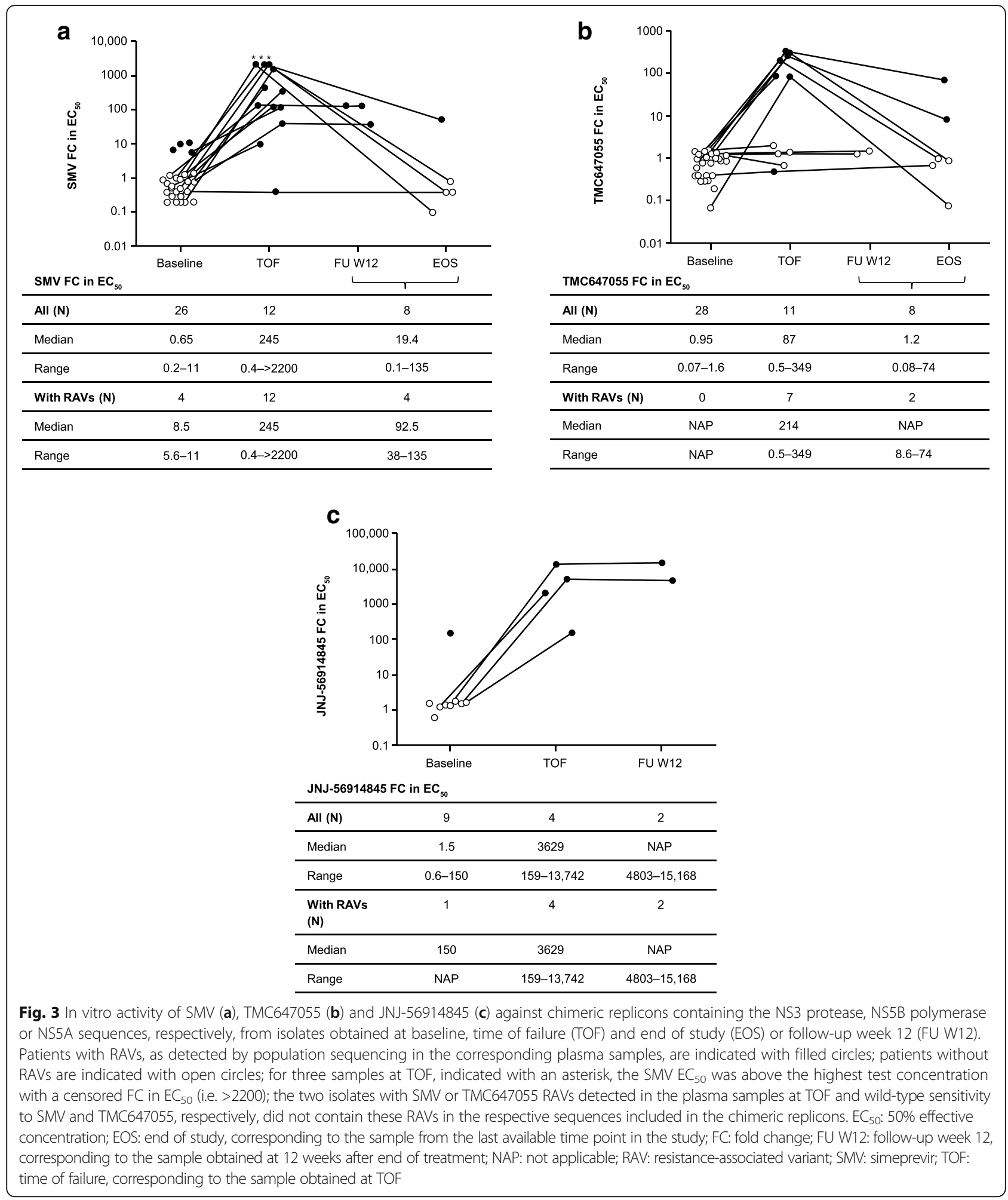

failure were not detected at baseline by deep sequencing in the set of samples analysed.

For all three inhibitors, a reduction in in vitro activity was observed against time of failure isolates that contained SMV, TMC647055 or JNJ-56914845 RAVs (Fig. 3). Isolates collected at time of failure, with no TMC647055 RAVs detected, remained fully susceptible to $\mathrm{TMC} 647055$ ( $\mathrm{FC}$ in $\mathrm{EC}_{50} \leq 2.0$ ). 
Table 1 Population sequencing and deep sequencing data for patients with virologic failure

\begin{tabular}{|c|c|c|c|c|c|c|c|c|c|}
\hline \multirow[t]{2}{*}{ Pt } & \multirow[t]{2}{*}{ GT } & \multirow[t]{2}{*}{ Reason for failure } & \multirow[t]{2}{*}{ Gene } & \multicolumn{2}{|l|}{ Baseline $^{a}$} & \multicolumn{2}{|l|}{ Time of failure ${ }^{a}$} & \multicolumn{2}{|l|}{ End of study ${ }^{a}$} \\
\hline & & & & PS & DS & $\overline{\mathrm{PS}^{\mathrm{b}}}$ & DS & $\overline{\mathrm{PS}^{\mathrm{b}}}$ & DS \\
\hline \multicolumn{10}{|c|}{ Panel 1} \\
\hline \multirow[t]{3}{*}{1} & GT1a & Viral breakthrough & NS3 & None & None & $\underline{\text { D168V }}$ & D168F 48.7\% & $\underline{\mathrm{R} 155 \mathrm{~K}}$ & R155K 7.8\% \\
\hline & & & & & & & D168V 50.4\% & & D168E $7.1 \%$ \\
\hline & & & NS5B & None & None & P495L & P495A 99.3\% & $\underline{\mathrm{P} 495 \mathrm{~L}}$ & P495L 29.0\% \\
\hline \multirow[t]{4}{*}{2} & GT1a & Viral breakthrough & NS3 & None & $\mathrm{S} 122 \mathrm{G} 1.7 \%$ & $\underline{D 168 \mathrm{~V}}$ & Q80R 60.8\% & $\underline{\mathrm{R} 155 \mathrm{~K}}$ & S122T $2.5 \%$ \\
\hline & & & & & & & R155K 61.6\% & & $\underline{\mathrm{R} 155 \mathrm{~K}} 98.9 \%$ \\
\hline & & & & & & & D168V 37.4\% & & \\
\hline & & & NS5B & None & None & P495L & P495L 99.5\% & None & P495L 13.6\% \\
\hline \multirow[t]{2}{*}{3} & GT1a & Viral breakthrough & NS3 & None & NA & $\underline{\mathrm{Q} 80 \mathrm{R}}+\underline{\mathrm{R} 155 \mathrm{~K}}$ & NA & $\underline{\mathrm{R} 155 \mathrm{~K}}$ & NA \\
\hline & & & NS5B & $1424 V+$ A499T & NA & $1424 \mathrm{~V}+\mathrm{A} 499 \mathrm{~T}+\underline{\mathbf{P 4 9 5 L}}$ & NA & $1424 V+$ A499T & NA \\
\hline \multirow[t]{3}{*}{4} & GT1a & Viral breakthrough & NS3 & Q80K & Q80K 99.1\% & $\underline{\mathrm{Q} 80 \mathrm{~K}}+\underline{\mathrm{R} 155 \mathrm{~K}}$ & NA & $\mathrm{Q} 80 \mathrm{~K}$ & Q80K 99.2\% \\
\hline & & & NS5B & L392F & L392F 99.7\% & L392F/L + P495L & NA & L392F & L392F 99.4\% \\
\hline & & & & & & & & & P495L 1.4\% \\
\hline \multirow[t]{5}{*}{5} & GT1a & Viral breakthrough & NS3 & Q80K & Q80K 98.6\% & $\underline{\mathrm{Q} 80 \mathrm{~K}}+\underline{\mathrm{R} 155 \mathrm{~K}}$ & NA & $\underline{\mathrm{Q} 80 \mathrm{~K}}+\underline{\mathrm{D} 168 \mathrm{E}}$ & Q80K 99.4\% \\
\hline & & & & & & & & & R155K 74.2\% \\
\hline & & & & & & & & & D168E 24.6\% \\
\hline & & & NS5B & V37I & V37I 99.4\% & $V 37 I+\underline{\text { P495L/S }}$ & NA & $V 371+\underline{\mathbf{P} 495 A}$ & V37I 98.2\% \\
\hline & & & & & & & & & P495A 32.5\% \\
\hline \multirow[t]{2}{*}{6} & GT1a & Late viral relapse ${ }^{c}$ & NS3 & None & None & $\underline{D 168 A}$ & ND & ND & ND \\
\hline & & & NS5B & None & А499T 1.6\% & None & ND & ND & ND \\
\hline
\end{tabular}

\section{Panel 2}

7 GT1b Viral breakthrough NS3 S122T

$\begin{array}{ll}\text { S122T 92.8\% } & \text { S122T }+\underline{\text { D168V }} \\ \text { None } & \underline{\text { P495L }} \\ \text { None } & \text { D168A/V }\end{array}$

8 GT1b Viral breakthrough NS3 None

None D168A/V

\begin{tabular}{|c|c|c|}
\hline NA & $\mathrm{S} 122 \mathrm{~T}$ & S122T 99.1\% \\
\hline NA & None & None \\
\hline D168A 63.6\% & None & None \\
\hline \multicolumn{3}{|l|}{ D168T 2.2\% } \\
\hline \multicolumn{3}{|l|}{ D168V 33.3\% } \\
\hline P495L 78.3\% & P495L & P495L 99.0\% \\
\hline \multicolumn{3}{|l|}{ P495S 20.0\% } \\
\hline A156V 99.8\% & None & None \\
\hline P495L 79.1\% & None & None \\
\hline NA & None & None \\
\hline NA & V499A & V499A 99.4\% \\
\hline D168V 99.8\% & None & None \\
\hline P495S 99.5\% & None & None \\
\hline
\end{tabular}

Panel 3

\begin{tabular}{|c|c|c|c|c|c|c|c|c|}
\hline GT1a & Detectable at EOT & NS3 & None & ND & $\underline{\mathrm{Q} 80 \mathrm{R}}+\underline{\mathrm{R} 155 \mathrm{~K}}$ & ND & Q80R & ND \\
\hline & & NS5B & None & ND & P495L & ND & None & ND \\
\hline GT1b & Viral breakthrough & NS3 & None & ND & D168V & ND & $\underline{\text { D168V }}$ & ND \\
\hline & & NS5B & None & ND & P495S & ND & None & ND \\
\hline GT1b & Detectable at EOT & NS3 & $\underline{\mathrm{Q} 80 \mathrm{R}}$ & ND & $\underline{\mathrm{Q} 80 \mathrm{R}}+\underline{\mathrm{D} 168 \mathrm{E}}$ & ND & $\underline{\mathrm{Q} 80 \mathrm{R}}+\underline{\mathrm{D} 168 \mathrm{E}}$ & ND \\
\hline & & NS5B & $1424 V+V 499 T$ & ND & $1424 \mathrm{~V}+\mathrm{V} 499 \mathrm{~T}+\underline{\mathrm{P} 495 \mathrm{~S}}$ & ND & $1424 V+V 499 T+\underline{P 495 S}$ & ND \\
\hline 15 GT1b & Viral relapse & NS3 & None & ND & D168V & ND & $\underline{D 168 \mathrm{~V}}$ & ND \\
\hline & & NS5B & V371+ 1424V & ND & V37I+ $1424 V+$ P495L & ND & $V 37 I+1424 V+$ P495L & ND \\
\hline
\end{tabular}


Table 1 Population sequencing and deep sequencing data for patients with virologic failure (Continued)

\begin{tabular}{|c|c|c|c|c|c|c|c|c|c|}
\hline \multirow[t]{2}{*}{16} & GT1b & Viral relapse & NS3 & S122T & ND & $\mathrm{S} 122 \mathrm{~T}+\underline{\mathrm{D} 168 \mathrm{~V}}$ & ND & $\mathrm{S} 122 \mathrm{~T}$ & ND \\
\hline & & & NS5B & None & ND & P495L & ND & None & ND \\
\hline \multirow[t]{2}{*}{17} & GT1b & Late viral relapse $^{c}$ & NS3 & None & ND & D168A & ND & ND & ND \\
\hline & & & NS5B & V37I & ND & V371 & ND & ND & ND \\
\hline \multicolumn{10}{|c|}{ Panel 4} \\
\hline \multirow[t]{6}{*}{18} & GT1a & Viral breakthrough & NS3 & None & None & $\underline{\mathrm{R} 155 \mathrm{~K}}$ & Q80R 9.3\% & $\underline{\mathrm{R} 155 \mathrm{~K}}$ & R155K 79.6\% \\
\hline & & & & & & & R155K 99.5\% & & \\
\hline & & & NS5B & $1424 \mathrm{~V}$ & L392F 3.6\% & $1424 V+\underline{P 495 L}$ & 1424L 2.5\% & $1424 \mathrm{~V}$ & 1424V 81.7\% \\
\hline & & & & & I424V 98.3\% & & I424V 92.2\% & & V494A $2.1 \%$ \\
\hline & & & & & & & P495L 85.2\% & & \\
\hline & & & NS5A & None & M28V 31.3\% & Q30E & Q30E 99.2\% & Q30E & Q30E 99.3\% \\
\hline \multirow[t]{3}{*}{19} & GT1a & Viral relapse & NS3 & None & None & Q80R & Q80R 99.0\% & Q80R & Q80R 99.7\% \\
\hline & & & NS5B & None & None & None & None & None & None \\
\hline & & & NS5A & None & None & Q30R & Q30R 99.1\% & Q30R & Q30R 99.6\% \\
\hline \multirow[t]{6}{*}{20} & GT1a & Viral relapse & NS3 & $\mathrm{S} 122 \mathrm{~T}$ & S122T 19.6\% & $\mathrm{S} 122 \mathrm{~T}+\underline{\mathbf{R} 155 \mathrm{~K}}$ & S122T 99.7\% & $\mathrm{S} 122 \mathrm{~T}+\underline{\mathbf{R} 155 \mathrm{~K}}$ & S122T 98.0\% \\
\hline & & & & & & & R155K 99.1\% & & R155K 95.5\% \\
\hline & & & NS5B & None & None & None & None & None & None \\
\hline & & & NS5A & None & None & $\underline{\mathrm{Q} 30 \mathrm{H}}+\underline{\mathrm{L} 31 \mathrm{M}}$ & Q30E 2.0\% & $\underline{\mathrm{Q} 30 \mathrm{H}}+\underline{\mathrm{L} 31 \mathrm{M}}$ & $\underline{\mathrm{Q} 30 \mathrm{H}} 98.3 \%$ \\
\hline & & & & & & & $\underline{\mathrm{Q} 30 \mathrm{H}} 93.6 \%$ & & L31M 98.6\% \\
\hline & & & & & & & L31M 94.1\% & & \\
\hline \multirow[t]{3}{*}{$21^{d}$} & GT1a & Viral relapse & NS3 & None & None & $\underline{\mathrm{R} 155 \mathrm{~K}}$ & R155K 99.4\% & $\underline{\mathrm{R} 155 \mathrm{~K}}$ & R155K 99.5\% \\
\hline & & & NS5B & None & None & None & None & None & None \\
\hline & & & NS5A & None & None & Q30E & Q30E 99.0\% & Q30E & Q30E 99.5\% \\
\hline \multirow[t]{4}{*}{22} & GT1a & Viral relapse & NS3 & $\underline{\mathrm{Q} 80 \mathrm{~K}}$ & Q80K 97.7\% & $\underline{\mathrm{Q} 80 \mathrm{~K}}+\underline{\mathrm{R} 155 \mathrm{~S}}$ & Q80K 99.8\% & $\underline{\mathrm{Q} 80 \mathrm{~K}}$ & Q80K 97.0\% \\
\hline & & & & & & & R155S 99.8\% & & $\underline{\mathrm{R} 155 \mathrm{~S}} 8.2 \%$ \\
\hline & & & NS5B & None & None & P495L & P495L 99.6\% & P495L & P495L 85.9\% \\
\hline & & & NS5A & $\mathrm{M} 28 \mathrm{~V}$ & M28V 23.3\% & $\underline{\mathrm{L} 31 \mathrm{M}}$ & L31M 99.7\% & $\underline{\mathrm{L} 31 \mathrm{M}}$ & M28A 9.4\% \\
\hline
\end{tabular}

${ }^{a}$ Amino acid substitutions are described considering six NS3 positions of interest $(43,80,122,155,156$ and 168), 18 NS5B positions of interest (37, 392, 393, 395, $396,399,424,425,428,429,492,493,494,495,496,499,500$ and 503 ) and five NS5A positions of interest (28, 30, 31, 32 and 93). RAVs (i.e. amino acid substitutions, when introduced as SDM in a GT1a or GT1b replicon, associated with a fold change in $\mathrm{EC}_{50}>2.0$ ) are underlined. Absence of amino acid substitutions considering the positions of interest is indicated with 'none'. DS data are reported when available for all genes of interest at a specific time point. ${ }^{b} \mathrm{Amino}$ acid substitutions shown in bold indicate emerging amino acid substitutions compared to baseline, based on PS. 'Late viral relapse defined as subject with SVR12 but with HCV RNA $\geq 25$ IU/mL at follow-up week 24 visit, the last scheduled visit in the study. ${ }^{\mathrm{d}}$ For subject 21 , end of study DS data were not available, instead data from the follow-up week 12 visit are shown

DS deep sequencing, $E C_{50} 50 \%$ effective concentration, EOT end of treatment, GT genotype, $H C V$ hepatitis $C$ virus, NA not available (no DS data available due to failure of amplification or Illumina DS reaction), ND not determined, PS population sequencing, Pt patient, RAV resistance-associated variant, SDM site-directed mutant, SVR12 sustained virologic response 12 weeks after actual end of treatment

\section{Persistence of emerging RAVs}

In $8 / 20$ patients $(40 \%)$ with emerging SMV RAVs at time of failure and follow-up NS3 sequencing data available, these SMV RAVs were no longer observed by population sequencing at end of study (Table 1). The median time between time of failure and end of study NS3 sequence was 20.6 weeks (range: $4.7-34.1$ weeks) and 25.5 weeks (range: 4.1-28.1 weeks), respectively, for the eight patients without and 12 patients with emerging SMV RAVs detected at end of study. Ten out of 16 patients (62.5\%) with emerging TMC647055 RAVs at time of failure and follow-up NS5B sequencing data available had lost these mutations at end of study, as assessed by population sequencing. The median time between time of failure and end of study NS5B sequence was 25.8 weeks (range: 20.1-28.4 weeks) and 23.9 weeks (range: 4.1-34.1 weeks), respectively, for the 10 patients without and the six patients with emerging TMC647055 RAVs detected at end of study. For all five patients with emerging JNJ56914845 RAVs at time of failure, these RAVs were still observed at end of study, with a median time between time of failure and end of study NS5A sequence of 20.1 weeks (range: 0-26.6 weeks). In the majority of patients with emerging RAVs no longer observed at 
end of study by population sequencing, these were also not detected by deep sequencing. In four patients (patients 2, 4, 18, 21), emerging TMC647055 or SMV RAVs were still detected by deep sequencing (read frequency $<25 \%$ ), while no longer present based on population sequencing.

In vitro susceptibility to the respective drugs was reduced when SMV, TMC647055 or JNJ-56914845 RAVs were still observed by population sequencing at end of study or follow-up week 12 (in case end of study isolate was not tested) (Fig. 3). When these RAVs were no longer detected by population sequencing, including in two patients (patients 2 and 4) with TMC647055 RAVs still detected by deep sequencing (read frequency $<25 \%$ ), wild-type sensitivity to the respective drugs was found.

\section{Discussion}

In this study, pre-existing RAVs were identified at low frequency. Baseline SMV RAVs were observed in $6.7 \%$ of patients with NS3 sequencing data available, and included Q80K in $5.6 \%$ of GT1-infected patients and in 9.1\% of GT1a-infected patients. In the SMV/pegIFN/ RBV Phase 2b/3 studies, the baseline prevalence of Q80K was higher (13.6\% in GT1-infected patients; $29.5 \%$ in GT1a-infected patients), which can be explained by the fact that the global Phase $2 b / 3$ studies included North America, a region with a high GT1a prevalence and a high Q80K prevalence within GT1a, while study TMC647055HPC2001 was performed in Europe [6]. In the SMV/pegIFN/RBV Phase $2 b / 3$ studies, SVR rates were substantially reduced in GT1a-infected patients with baseline Q80K compared to those without this polymorphism [23-25]. The two GT1a-infected patients with baseline $\mathrm{Q} 80 \mathrm{~K}$ in the 2-DAA regimen did not achieve SVR; however, virologic failure rates were generally high (6/10) in Panel 1 . In the 3-DAA regimen, one of the two GT1a-infected patients with baseline Q80K achieved SVR. Baseline JNJ-56914845 RAVs were present in $4.8 \%(2 / 42)$ of patients with NS5A sequencing data available; both patients achieved SVR. However, the low number of patients with baseline RAVs did not allow conclusions to be drawn on the impact of the presence of baseline RAVs on treatment outcome.

In the majority of patients with virologic failure, emerging RAVs to all study drugs were observed at time of failure. Similar findings of emerging resistance to multiple classes of DAAs have been described in patients with virologic failure in other 12 -week combination regimens of DAAs with similar mechanisms of action i.e. an NS3/4A protease inhibitor, paritaprevir/ritonavir, combined with an NNI, dasabuvir, with or without an NS5A inhibitor, ombitasvir [26, 27]. All patients had SMV RAVs at time of failure, detected at NS3 positions 80, 155, 156 and/or 168, consistent with the previously characterised SMV resistance profile $[6,7,9,10,28]$. Emerging TMC647055 RAVs were found at time of failure in the majority of patients (in $14 / 17$ and $2 / 5$ patients with virologic failure in the 2- and 3-DAA regimens, respectively). These were observed at NS5B position 495 only, similar to the findings from in vitro TMC647055 selection experiments [11] and from the Phase 1b study that evaluated TMC647055 in monotherapy and in combination with SMV $[12,13]$. There was no difference found in emerging RAVs between a low dose of TMC647055/RTV (450 mg QD; Panels 1-2) and a high dose (600 mg QD; Panel 3) or between HCV geno/subtypes. All five 3-DAA failure patients had emerging JNJ56914845 RAVs at NS5A positions 30 and/or 31. RAVs at these positions were also identified in JNJ-56914845 in vitro selection experiments and in the Phase 1 monotherapy study $[14,15]$. The RAVs observed at time of failure were associated with a $>100$-fold reduction in JNJ-56914845 in vitro activity [14]. No in vitro JNJ56914845 susceptibility data were available for Q30E; however, from data available from other NS5A inhibitors, it can be expected that this mutation also confers resistance to JNJ-56914845 [29]. Illumina deep sequencing, performed for a subset of time of failure isolates, confirmed the absence of TMC647055 RAVs in patients without these RAVs detected by population sequencing. For the selected set of samples, none of the RAVs emerging at time of failure were found to be already present at baseline by using deep sequencing.

While emerging SMV and TMC647055 RAVs became undetectable by population sequencing at end of study in $40 \%(8 / 20)$ and $62.5 \%(10 / 16)$ of patients, respectively, emerging JNJ-56914845 RAVs were still observed at the end of study in all five 3-DAA failure patients. These observations confirm earlier findings of disappearance of emerging SMV RAVs in half of the patients at end of study in SMV/pegIFN/RBV studies [6] (as assessed by population sequencing), as well as the long-term persistence of emerging NS5A RAVs that has been demonstrated in patients who failed ledipasvir-containing regimens [30].

In the majority of patients in whom emerging RAVs in the NS3 and NS5B regions were no longer observed at end of study by population sequencing, these were also no longer detected by the more sensitive Illumina deep sequencing technology, in line with findings from previous studies [31, 32].

Phenotypic analysis showed a reduction in sensitivity to the study drugs when RAVs were detected by population sequencing. Consistency between the detection of SMV RAVs in clinical isolates and their in vitro susceptibility to SMV was also demonstrated in a recent study investigating clinical isolates of $\mathrm{HCV}$ GT1-infected patients enrolled in SMV Phase 1-3 clinical studies [20]. 


\section{Conclusions}

Virologic failure in study TMC647055HPC2001 was associated with the emergence of RAVs to all study drugs in the majority of patients. Treatment-emergent SMV and TMC647055 RAVs became undetectable over time in many of the patients after treatment was stopped, while emerging JNJ-56914845 RAVs could still be detected at end of study in the five 3-DAA failure patients.

\section{Additional file}

Additional file 1: Study design. HCV NS3/4A, NS5B and NS5A sequence analysis. (DOCX $32 \mathrm{~kb}$ )

\section{Abbreviations}

BID: Twice daily; DAA: Direct-acting antiviral; $\mathrm{EC}_{50}$ : 50\% effective concentration; EU: European Union; FC: Fold change; GT: Genotype; HCV: Hepatitis C virus; IFN: Interferon; NAP: Not applicable; ND: Not determined; NNI: Non-nucleoside inhibitor; pegIFN: Pegylated interferon; QD: Once daily; RAV: Resistance-associated variant; RBV: Ribavirin; RTV: Ritonavir; SDM: Site-directed mutant; SMV: Simeprevir; SVR12: Sustained virologic response 12 weeks after actual end of treatment; US: United States

\section{Acknowledgements}

The authors would like to thank the patients and investigators for their contributions to this study. Medical writing support was provided by Luc Geeraert of Bench-to-Pen and by Chrissie Kouremenou of Complete Medical Communications, and funded by Janssen. Additional statistical and programming support was provided by Hilde Wuyts, Moshine El Ghazi and Gert Van Beek.

\section{Funding}

The study was funded by Janssen. Janssen were responsible for the study design and the collection and interpretation of data. Medical writing support was funded by Janssen.

\section{Availability of data and materials}

The datasets generated during and/or analysed during the current study are available from the corresponding author on reasonable request.

\section{Authors' contributions}

$L V, K T, A V, P V R, R V$, and SDM were involved in the study concept and design and the acquisition, analysis and interpretation of study data. They have also been involved in the critical revision of the manuscript for important intellectual content. All authors read and approved the final manuscript.

\section{Competing interests}

$L V, K T, A V, P V R$, and SDM are employees of Janssen and may own stock in Johnson \& Johnson. RV was an employee of Janssen at the time this study took place.

\section{Consent for publication}

Not applicable.

\section{Ethics approval and consent to participate}

The study was approved by the Institutional Review Board or Independent Ethics Committee at each participating centre, and met the ethical principles of the Declaration of Helsinki and Good Clinical Practice guidelines. All patients provided written, informed consent.

\section{Publisher's Note}

Springer Nature remains neutral with regard to jurisdictional claims in published maps and institutional affiliations.
Received: 5 December 2016 Accepted: 1 May 2017

Published online: 31 May 2017

\section{References}

1. World Health Organization. WHO Fact Sheet 164 - Hepatitis C 2012. http:// www.who.int/mediacentre/factsheets/fs164/en/. Accessed 23 Nov 2016.

2. Lam BP, Jeffers T, Younoszai Z, Fazel Y, Younossi ZM. The changing landscape of hepatitis C virus therapy: focus on interferon-free treatment. Therap Adv Gastroenterol. 2015:8:298-312.

3. Pawlotsky JM, Feld J, Zeuzem S, Hoofnagle JH. From non-A, non-B hepatitis to hepatitis C virus cure. J Hepatol. 2015:62:S87-99.

4. Hezode C, Bronowicki JP. Ideal oral combinations to eradicate HCV: The role of ribavirin. J Hepatol. 2016:64:215-25.

5. Bourgeois S, Van Vlierberghe H, Moreno C, Orlent H, Nevens F, Arasteh K, et al. Efficacy, safety and pharmacokinetics of simeprevir and TMC647055/ritonavir with or without ribavirin and JNJ-56914845 in HCV genotype 1 infection. BMC Gastroenterol. 2017;17:26

6. Lenz $\mathrm{O}$, Verbinnen $\mathrm{T}$, Fevery $\mathrm{B}$, Tambuyzer L, Vijgen $\mathrm{L}$, Peeters $\mathrm{M}$, et al. Virology analyses of HCV isolates from genotype 1-infected patients treated with simeprevir plus peginterferon/ribavirin in Phase IIb/III studies. J Hepatol. 2015;62:1008-14

7. Lawitz E, Sulkowski MS, Ghalib R, Rodriguez-Torres M, Younossi ZM, Corregidor A, et al. Simeprevir plus sofosbuvir, with or without ribavirin, to treat chronic infection with hepatitis $C$ virus genotype 1 in non-responders to pegylated interferon and ribavirin and treatment-naive patients: the COSMOS randomised study. Lancet. 2014;384:1756-65.

8. Moreno C, Hezode C, Marcellin P, Bourgeois S, Francque S, Samuel D, et al. Efficacy and safety of simeprevir with PegIFN/ribavirin in naive or experienced patients infected with chronic HCV genotype 4. J Hepatol. 2015;62:1047-55.

9. Lawitz E, Matusow G, DeJesus E, Yoshida EM, Felizarta F, Ghalib R, et al. Simeprevir plus sofosbuvir in patients with chronic hepatitis $C$ virus genotype 1 infection and cirrhosis: a phase 3 study (OPTIMIST-2). Hepatology. 2016;64:360-9.

10. Kwo P, Gitlin N, Nahass R, Bernstein B, Rojter S, Schiff E, et al. Simeprevir plus sofosbuvir (12 and 8 weeks) in HCV genotype 1-infected patients without cirrhosis: OPTIMIST-1, a phase 3, randomized study. Hepatology. 2016;64:370-80

11. Devogelaere B, Berke JM, Vijgen L, Dehertogh P, Fransen $E$, Cleiren E, et al. TMC647055, a potent nonnucleoside hepatitis C virus NS5B polymerase inhibitor with cross-genotypic coverage. Antimicrob Agents Chemother. 2012:56:4676-84.

12. Leempoels J, Reesink H, Bourgeois S, Vijgen L, Rouan MC, Vandebosch A, et al. Human safety, pharmacokinetics and antiviral activity of TMC647055, a novel HCV non-nucleoside polymerase inhibitor. 2011. http://www.natap. org/2011/AASLD/AASLD 04.htm. Accessed 23 Nov 2016.

13. Bourgeois S, Reesink HW, Leempoels J, Vijgen L, Rouan MC, Marien K, et al Combination therapy of TMC647055 with simeprevir (TMC435) in chronic hepatitis C patients [abstract]. J Hepatol. 2013;58:S483.

14. Walker J, Crosby R, Wang A, Woldu E, Vamathevan J, Voitenleitner C, et al. Preclinical characterization of GSK2336805, a novel inhibitor of hepatitis C virus replication that selects for resistance in NS5A. Antimicrob Agents Chemother. 2014:58:38-47.

15. Wilfret DA, Walker J, Adkison KK, Jones LA, Lou Y, Gan J, et al. Safety, tolerability, pharmacokinetics, and antiviral activity of GSK2336805, an inhibitor of hepatitis C virus (HCV) NS5A, in healthy subjects and subjects chronically infected with HCV genotype 1. Antimicrob Agents Chemother. 2013:57:5037-44.

16. Koletzki D, Pattery T, Fevery B, Vanhooren L, Stuyver LJ. Amplification and sequencing of the hepatitis C virus NS3/4A protease and the NS5B polymerase regions for genotypic resistance detection of clinical isolates of subtypes 1a and 1b. Methods Mol Biol. 2013:1030:137-49.

17. Thys K, Verhasselt P, Reumers J, Verbist BM, Maes B, Aerssens J. Performance assessment of the Illumina massively parallel sequencing platform for deep sequencing analysis of viral minority variants. J Virol Methods. 2015;221:29-38.

18. Nyanguile $O$, Devogelaere B, Vijgen L, Van den Broeck W, Pauwels F, Cummings MD, et al. 1a/1b subtype profiling of nonnucleoside polymerase inhibitors of hepatitis C virus. J Virol. 2010;84:2923-34.

19. Vijgen L, Verbeeck J, van Kerckhove B, Berke JM, Koletzki D, Fanning G, et al. A cellular replicon-based phenotyping assay to determine susceptibility of hepatitis C virus clinical isolates to NS3/4A protease inhibitors. Methods Mo Biol. 2013;1030:105-17.

20. Verbinnen T, Fevery B, Vijgen L, Jacobs T, De Meyer S, Lenz O. In vitro activity of simeprevir against hepatitis $C$ virus genotype- 1 clinical isolates and its 
correlation with NS3 sequence and site-directed mutants. Antimicrob Agents Chemother. 2015;59:7548-57.

21. Huang W, Newton A, Cook J, Toma J, Frantzell A, Choe S, et al. Development of recombinant replicon report assays for characterization of HCV NS5A and NS3/4A protease inhibitors. Antiviral Ther. 2011;16(Suppl. 1): A29.

22. Lenz O, Verbinnen T, Lin T-I, Vijgen L, Cummings MD, Lindberg J, et al. In vitro resistance profile of the hepatitis $C$ virus NS3/4A protease inhibitor TMC435. Antimicrob Agents Chemother. 2010;54:1878-87.

23. Fried MW, Buti M, Dore GJ, Flisiak R, Ferenci P, Jacobson I, et al. Once-daily simeprevir (TMC435) with pegylated interferon and ribavirin in treatmentnaive genotype 1 hepatitis C: the randomized PILLAR study. Hepatology. 2013;58:1918-29.

24. Forns X, Lawitz E, Zeuzem S, Gane E, Bronowicki JP, Andreone P, et al. Simeprevir with peginterferon and ribavirin leads to high rates of SVR in patients with HCV genotype 1 who relapsed after previous therapy: a phase 3 trial. Gastroenterology. 2014;146:1669-79.

25. Jacobson IM, Dore GJ, Foster GR, Fried MW, Radu M, Rafalskiy W, et al. Simeprevir with pegylated interferon alfa 2a plus ribavirin in treatment-naive patients with chronic hepatitis C virus genotype 1 infection (QUEST-1): a phase 3, randomised, double-blind, placebo-controlled trial. Lancet. 2014;384:403-13.

26. Poordad F, Lawitz E, Kowdley KV, Cohen DE, Podsadecki T, Siggelkow S, et al. Exploratory study of oral combination antiviral therapy for hepatitis C. N Engl J Med. 2013;368:45-53.

27. Krishnan P, Tripathi R, Schnell G, Reisch T, Beyer J, Irvin M, et al. Resistance analysis of baseline and treatment-emergent variants in hepatitis $C$ virus genotype 1 in the AVIATOR study with paritaprevir-ritonavir, ombitasvir, and dasabuvir. Antimicrob Agents Chemother. 2015;59:5445-54

28. Fevery B, Vijgen L, Van Eygen V, Jessner W, Corbett C, Schlag M, et al. Consistent simeprevir resistance profile in hepatitis $C$ virus genotype 1-infected patients failing simeprevir interferon-free compared with interferon-containing regimens [abstract]. J Hepatol. 2016;64:S399.

29. Sarrazin C. The importance of resistance to direct antiviral drugs in HCV infection in clinical practice. J Hepatol. 2016;64:486-504.

30. Wyles D, Mangia A, Cheng W, Shafran A, Schwabe C, Ouyang W, et al. Long-term persistence of HCV NS5A variants after treatment with NS5A inhibitor ledipasvir. J Hepatol. 2015;62(Suppl. 2):S221.

31. Fevery B, Thys K, Van Eygen V, Verbinnen T, Van Rossem E, Buelens A, et al. Pre-existence and persistence of resistant minority hepatitis $C$ virus variants in genotype 1-infected patients treated with simeprevir/peginterferon/ribavirin. Open Forum Infect Dis. 2016;3:ofw052.

32. Dierynck I, Thys K, Ghys A, Sullivan JC, Kieffer TL, Aerssens J, et al. Deepsequencing analysis of the gene encoding the hepatitis $C$ virus nonstructural 3-4A protease confirms a low prevalence of telaprevir-resistant variants at baseline and the end of the REALIZE study. J Infect Dis. 2014;210:1871-80.

\section{Submit your next manuscript to BioMed Central and we will help you at every step:}

- We accept pre-submission inquiries

- Our selector tool helps you to find the most relevant journal

- We provide round the clock customer support

- Convenient online submission

- Thorough peer review

- Inclusion in PubMed and all major indexing services

- Maximum visibility for your research

Submit your manuscript at www.biomedcentral.com/submit

) Biomed Central 